\title{
Comparative evaluation of the efficacy of different neurorehabilitation programs on the functional recovery and the autonomy of patients with post stroke hemiparesis
}

\author{
Ivet Koleva $^{1 *}$, Radoslav Yoshinov ${ }^{2}$ and Borislav Yoshinov ${ }^{1}$ \\ ${ }^{1}$ Medical University of Sofia, Bulgaria \\ ${ }^{2}$ Bulgarian Academy of Sciences - Sofia, Bulgaria
}

\begin{abstract}
Aim: On the base of systematic review of the literature, clinical observations and investigations, specific complex neurorehabilitation algorithms were structured (including natural and preformed physical modalities).

GOAL: Comparative evaluation of the efficacy of different physical-therapeutic and rehabilitation programs on the functional recovery, the autonomy in activities of daily living and the capacity for different activities of patients suffering from post-stroke hemiparesis.

Materials and methods: A total of 366 post-stroke in-patients were observed. The neurorehabilitation complex includes physiotherapy, occupational therapy; thermotherapy or cryotherapy; in some groups we added electrostimulations of hand extensors and mirror therapy (grasp training).

Patients were investigated before and after therapy, and one month later; according a Protocol with all patients' data (including neurological and functional status, hemiparesis level - Brunnstrom's test; evaluation of capacity of independence in self-care, familiar activities, professional activities; evaluation of prehension).

Statistical evaluation: $t$-test (analysis of variances ANOVA) and Wilcoxon rank test (non-parametrical correlation analysis).

Results and discussion: Comparative analysis of results proved a statistically significant favorable effect $(\mathrm{p}<0,001)$ on some parameters: increase of the muscle force, the range of motion and the functional capacity of the hand (precision grips); stabilization of the balance and the locomotion (test of Brunnstrom); improvement of the autonomy in different activities - self-service and domestic care. The stability of the results one month after the end of neurorehabilitation is satisfactory.
\end{abstract}

Conclusion: In conclusion we must underline the impact of electrostimulations and grasp training before mirror for functional recovery and patients' autonomy.

\section{INTRODUCTION}

Stroke is considered worldwide as a socially important disease, with high morbidity and mortality, and a significant level of disability of stroke survivors, with necessity of systematic rehabilitation [1-3].

According to the data of the American National Stroke Association: stroke is one of the leading causes of long-term adult disability, affecting approximately 795,000 people each year in the U.S. [1].

According to reports of the National Institute of Neurological Disorders and Stroke (NINDS), a component of the U.S. National Institutes of Health (NIH), approximately two-thirds of the stroke patients in the United States survive and require rehabilitation $[3,4]$.

According to the World Health Organization (WHO), stroke is a condition in which clinical signs of focal or global impairment of cerebral functions develops rapidly, lasts longer than 24 hours unless interrupted by surgery or death, and exhibit no apparent nonvascular cause [1]. From the clinical point of view, the acute neurological deficit is the result of a large area of impaired circulation. So, the stroke is a result of the altered circulation, respectively it can be defined as a vesselrelated functional impairment of a specific region of the brain. Strokes can be classified according to the type: ischemic stroke (80-83\%); intracerebral hemorrhage (10-12\%); subarachnoid hemorrhage (7-8\%).
About $88 \%$ of the patients with acute stroke have hemiparesis [2], so the motor weakness and the reduced autonomy in everyday life are the main problems of post stroke patients in rehabilitation units.

The World Health Organization's (WHO) definition of rehabilitation is: "The use of all means aimed to reduce the impact of disabling and handicapping conditions, and at enabling people with disabilities to achieve optimal social integration" [5,6].

The definition of Physical and Rehabilitation Medicine (PRM) by the Union Européenne des Médecins Spécialistes (European Union of Medical Specialists) (UEMS) - Section of PRM is: "an independent medical specialty concerned with the promotion of physical and cognitive functioning, activities (including behavior), participation (including quality of life) and modifying personal and environmental

Correspondence to: Prof. Dr Ivet Koleva, MD, PhD, D.Med.Sc, Medical University of Sofia - Bulgaria, Tel: ++359.888 2081 61; E-mail: yvette@cc.bas.bg

Key words: neurorehabilitation, stroke, hemiparesis, algorithm, functional recovery, activities of daily living $(A D L)$

Received: September 28, 2016; Accepted: November 29, 2016; Published: December 03, 2016 
Koleva I (2016) Comparative evaluation of the efficacy of different neurorehabilitation programs on the functional recovery and the autonomy of patients with post stroke hemiparesis

factors. It is thus responsible for the prevention, diagnosis, treatments and rehabilitation management of people with disabling medical conditions and co-morbidity across all ages." [5].

There is not a great difference of basic concepts in PRM between both sides of the Atlantic [7].

According to these concepts, the main objective of rehabilitation is to help survivors become as independent as possible and to attain the best possible quality of life [NINDS], and stroke is the second neurological typical condition present in acute PRM wards [6].

Physical and Rehabilitation Medicine (PRM) is an independent medical specialty focusing on the improvement of functioning [8], and the role of International Classification of Functioning, disability and Health $[\mathrm{ICF}]$ is crucial $[9,10]$. During last two decades we worked on this [11].

Rehabilitation in post-stroke patients traditionally applies different PRM techniques: Proprioceptive neuromuscular facilitation, Kabath and Brunnstrom methods; Neurodevelopmental training (Bobath concept); Sensorimotor therapy (Rood approach). Ultimately some modern modalities were included in the neurorehabilitation process, such as Motor relearning program, Constraint-induced movement therapy, Mirror therapy, Functional electrical stimulation (FES), Electromyographic (EMG) biofeedback, Robotic devices $[2,11,12]$.

Most of the studies analyze the isolated efficacy of one or two rehabilitation methods. But medical doctors - PRM specialists traditionally have a holistic approach to people with acute and chronic conditions [5]. Thus, we decide to combine different physical modalities (natural and pre-formed), with the goal to improve the efficacy of the neurorehabilitation [11]. In our clinical practice, we accentuate on the functional recovery, capacities for different activities and autonomy in everyday life, and we apply a synergic combination of physiotherapy, occupational therapy, thermo- or cryotherapy, electrotherapy, etc.

\section{GOAL}

The goal of the current article is to effectuate a comparative evaluation of the efficacy of application of different neurorehabilitation programs in patients with post-stroke hemiparesis, and to evaluate the impact of different physical modalities: physiotherapy (proprioceptive neuro-muscular facilitation techniques, passive and active range-ofmotion exercises, gait training), mirror therapy for digital prehension training, occupational therapy, cryotherapy or thermotherapy, functional electrostimulations.

\section{DESIGN OF THE STUDY (MATERIAL AND METHODS)}

The current investigation was realized during last 15 years in 5 university departments / clinics of PRM in two Bulgarian cities (the capital Sofia and one city at the north part of the country - Pleven).

The investigations were carried out considering patients' rights (according to the Declaration of Helsinki), and were approved by the respective Institutional review boards and Ethic commissions.

All patients signed informed consent concerning their participation in the scientific research, and before undergoing every investigation or procedure.

In the investigation we include a total of 366 in-patients, treated using 6 different neurorehabilitation complexes. For controls we used data of patients without PRM (included in the list of potential inpatients, during the interval between the initial examination and their entry in the Neurorehabilitation Clinic). All patients were examined by us, during our work or consultation.

Inclusion criteria: The clinical diagnosis of all patients was post-stroke hemiparesis; proved by clinical, functional and imagery examinations.

Exclusion criteria: All patients with a severe cardiac, respiratory or renal insufficiency were excluded, general contraindications for rehabilitation were considered. We excluded patients with correction of the medicaments during the last 1 month before the beginning of our study.

Characteristics of the material: Our patients were in a subacute or chronic stage after a supra-tentorial stroke; presenting a spastic hemiparesis (including central paresis of the ipsilateral facial and the hypoglossal nerve), with significant motor weakness, hyperreflexia, pathologic reflexes of the groups of Babinski and Rossolimo, superficial hemi-hypoesthesia; with difficulties in the functional grasping and transfers, and with reduced autonomy in everyday activities.

A total of 366 patients were treated, the distribution men: women $=151: 215(41,26 \%: 58.74 \%)$. The middle age of our patients was 57 years (from 34 to $68 \mathrm{y}$, standard deviation $/ \mathrm{SD} /=13,8$ ); in a period of 1 to 8 months (average 4,5 months, SD 3,8) after an ischemic stroke (cerebral infarctus) in the irrigation territory of the medial cerebral artery (left arteria cerebri media /ACM/ in 149 patients, right ACM in 117 patients). The Brunnstrom score before the neurorehabilitation was on a middle level III - IV, the evaluated functional capacity was 13 / 20 points (Barthel ADL index).

Previously, all patients were hospitalized in an University Clinic/ Department of Neurology.

Our intervention began during the first months after the acute event ( 1.5 to 8 months); and was realized on a total of 366 post-stroke in-patients of a Clinic/Department of Physical and Rehabilitation Medicine (Neurorehabilitation/neuroreh/sector).

Every patient received a complex PRM program of four weeks (20 procedures, 5 times weekly).

Periods of evaluation: Patients were controlled before and at the end of the neuroreh course, and one month later; according a Protocol with all data - using a battery of traditional and contemporaneous objective methods (including neurological and functional status): level of the hemiparesis - Brunnstrom's test; tests and scales for motor weakness, balance and coordination; tests for spasticity and prehension (functional grip); tests for gait and independent motion; functional scales for capacity and autonomy in daily living activities (self-service, family life, professional, social); scales for depression and anxiety (tests of Zung); etc.

Here we analyze results from all patients, which passed this program and who were presented to the control examination one month after the end of the rehabilitation.

Randomization of patients: In the investigation we included a total of 366 in-patients, divided in 7 groups: three experimental groups of a total of 285 patients treated by innovative physiotherapy methods (E-1, E-2, E-3), and 81 patients for the control groups: three of them treated by standard rehabilitation methods (K-1, K-2, K-3) and a control group patients without PRM (K-0). Patients were randomized into seven treatment groups (approximatively 95 patients per group - for the experimental groups; about 20 patients per group - for the control groups). 
Koleva I (2016) Comparative evaluation of the efficacy of different neurorehabilitation programs on the functional recovery and the autonomy of patients with post stroke hemiparesis

Functional assessment methods: The evaluation protocol consists of: the history of the condition, the clinical patterns of the central hemiparesis and the autonomy in everyday activities, and functional assessment, including: the level of the hemiparesis (Brunnstrom's test) [13], the functional score (Barthel ADL index - score of 20 points) [14], the evaluation of spasticity (0-4), the goniometry of the range of motion of the wrist and the evaluation of the functional capacities of the paretic upper limb in activities (test of E.Michel), evaluation of the autonomy in activities of daily living (0-5) - concerning self-care (bed activities, toilet, dressing, eating, transfers), domestic activities (fixing the bed, food preparation, doing the clothing, cleaning); professional activities (for the upper and lower extremities; professional activities connected with travelling); and evaluation (0-5) of the digital prehension or terminal opposition (precision grips - tip-to-tip pinch) [11].

\section{Treatment methods}

The neurorehabilitation program was focused on the following tasks: functional recovery, restoration of functional independence; reduction of muscle weakness and spasticity; training of transfers, grasp and gait; management of co-morbid conditions; prevention of complications (e.g. muscle and joint contractures, position and gait type Wernicke-Mann, humero-scapular sub-luxation); increase of the quality of life of patient and his / her family; social re-integration.

The duration of the PRM-program was 20 procedures, 5 days a week, for 4 weeks.

All patients received a detailed physiotherapeutic program, including proprioceptive neuro-muscular facilitation (PNF - method of Kabath), education and training of some activities for the self-care. Family members were included in the neurorehabilitation team and participated in the sessions of patients' education.

For all patients of the experimental groups the PRM programs included:

$>$ Physiotherapy: individualized movement program (PNF by Kabath) with soft tissue massages (proprioceptive neuro-muscular facilitation techniques, analytic exercises for the paretic limbs, balance training, training of transfers and gait, exercises to stabilize the gait on flat terrain and on stairs); duration 45 minutes, twice a day;

cryotherapy with ice block - distally in upper and lower extremities, duration 2-3 minutes per field, twice daily;

occupational therapy $(O T)$, including training in activities of daily living (ADL) - 30 minutes, twice a day;

\section{$>$ patient education.}

In the second group (E-2) we added:

$>$ functional electrical stimulations (FES) - a stable method of application, for the extensors of the paretic upper and lower limbs (with special attention to the wrist and ankle) and for the flexors of the fingers; with tetanic pulses, procedure of 10-20 minutes, once a day;

In the third group (E-3) we added too:

$>\quad$ analytic exercises and manipulative activities (grasp training with peanuts, beans, lego) with a mirror box (mirror therapy) - 10-20 minutes a day.

In the control groups $(K)$ we included a total of 81 patients (with comparable clinical characteristics), part of them - control zero group $(K-0)$ were observed without rehabilitation (between the initial examination before the entrance in the PRM clinic and the official entry like in-patients). The rest of patients of the control groups were randomized into three control groups (K-1, K-2, K-3) and they received a standard physiotherapy program (once daily) and a standard (group) education in some ADL.

○ $\quad$ K-1 sub-group received only these procedures;

- In patients of $\boldsymbol{K}-\mathbf{2}$ sub-group we added too thermotherapy (paraffin applications distally (wrist and ankle);

○ K-3 sub-group received additionally electrostimulations once a day.

All patients received detailed instructions for the treatment strategy and concerning the rehabilitation process. Patient education includes information about the lesion and the main risk factors for stroke, possible therapy and interventions, potential of the physical and rehabilitation medicine (PRM); and principal recommendations for everyday life during the first 6 months (position therapy, control of spasticity, grasp and gait training; hypolipid and hypoglucid diet; ending of smoking and alcohol abuse; control of lipid profile and arterial hypertension, stress control).

\section{Statistical analysis}

Statistical analysis was performed with SPSS electronic package, version 17. We applied options for two samples comparison with parametrical analysis of variances ANOVA and non-parametrical distribution and correlation analysis: $t$-test ( $t$-criteria, $p$ value), Signed test, Signed rank test, Kolmogorov - Smirnov test, Mann - Whitney (Wilcoxon) W test (W median).

The treatment difference was considered to be statistically significant if the $\mathrm{p}$ value was $<0.05$. In some cases we received lower results of the $\mathrm{p}$-value $(\mathrm{p}<0.01$ and even $\mathrm{p}<0.001)$.

\section{RESULTS}

Comparative analysis of results of neurological and functional exams proved a statistically significant favorable effect $(\mathrm{p}<0,05)$ on a lot of parameters: reduction of muscle weakness, increase of the range of motion and the functional capacity of the hand (including grip); stabilization of the balance and locomotion (tests of Brunnstrom); improvement of capacity of hemiparetics to effectuate different activities - self-care, domestic care. The stability of the results one month after the end of neurorehabilitation was satisfactory.

\section{Functional capacities}

The evaluation using the Brunnstrom's test [13] presents an increase of functional capacities. Table 1 compares the score before and after therapy (B.Th./A.Th.).

The statistical analysis of the total distribution of patients according the score of S Brunnstrom is presented on Figure 1. Using the method of Mann - Whitney we don't receive significant difference ( $p>0.05$ ), because of the gravity of the hemiparesis and the relatively short duration of the rehabilitation (according actual situation in our country). But we observed a translation of the curve of distribution (Gaus distribution) after rehabilitation to the left side (translation to the mild levels of alteration) - in comparison with the levels before therapy (B.Th.), especially for the patients of levels 3 and 4 .

The statistical analysis (t-test) of the results concerning the functional evaluation by the $A D L$ scale of Barthel (Figure 2) and the test 
Koleva I (2016) Comparative evaluation of the efficacy of different neurorehabilitation programs on the functional recovery and the autonomy of patients with post stroke hemiparesis

Table 1. Evaluation with the test of Signe Brunnstrom.

\begin{tabular}{|c|c|c|c|}
\hline Moment of the test & \multicolumn{2}{|c|}{ Before Neurorehabilitation } & \multicolumn{2}{c|}{ After Neurorehabilitation } \\
\hline Brunnstrom's score & Number of patients & Value per cent & Number of patients \\
\hline Level 2 & $\mathbf{1 1 7}$ & 41,06 & $\mathbf{2 6}$ \\
\hline Level 3 & $\mathbf{1 5 3}$ & 53,68 & $\mathbf{9 7}$ \\
\hline Level 4 & $\mathbf{1 5}$ & 5,26 & $\mathbf{9 1}$ \\
\hline Level 5 & $\mathbf{0}$ & 0 & $\mathbf{1 5 6}$ \\
\hline TOTAL & $\mathbf{2 8 5}$ & $\mathbf{1 0 0 . 0 0}$ & $\mathbf{6}$ \\
\hline
\end{tabular}

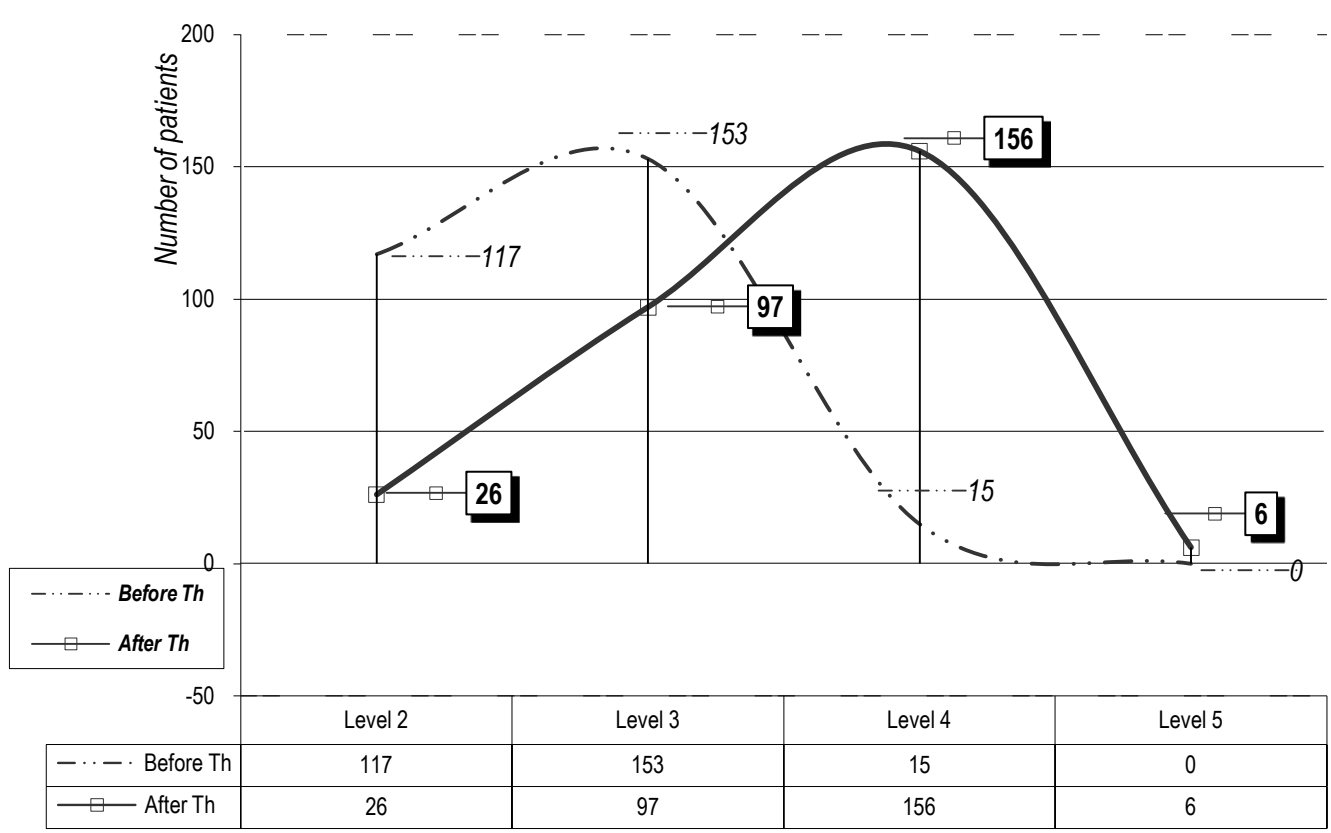

Figure 1. Functional evaluation (test of S Brunnstrom) (for the 285 patients of the experimental groups).

of Michels for the upper extremity (Figure 3) demonstrates a significant difference $(\mathrm{p}<0.05)$ for the patients of the experimental groups $(\mathrm{E}-1$, E-2 \& E-3), especially for the group E-3.

We must highlight that the results are more important for the patients with a mild level of dysfunction.

\section{Spasticity}

The evaluation of spasticity presents a favorable effect - reduction of spasticity (values A.Th. in comparison with the values before Th Table 2 and Figure 4).

\section{Independence in activities of daily living (ADL)}

We observed significant amelioration in autonomy of patients in $A D L(0-5)$ - concerning self-care and some domestic activities (Figure 5): bed activities, sitting, standing, balance; transfers - using or not a wheelchair; personal hygiene, dressing; eating). Our patients didn't present significant performance in home and professional activities - probably due to the level of motor weakness, the short duration of neurorehabilitation and the early period after the acute onset of the vascular event.

During the statistical analysis (t-test) of self-care activities (0-5) we observed median values: before therapy (B.Th.) $=1,33+/-\operatorname{SD~} 0,072$; and after therapy (A.Th.) $=2,15+/$ - SD 0,$768 ; \mathrm{p}=0,016$, so we have a statistical significance with a $\mathrm{p}<0.05$. The statistical significance is most pronounced for the patients of the experimental groups: E-1, E-2 and E-3. Applying the method of Mann-Whitney we received values for the $\mathrm{W}$-medians B.Th. and A.Th.: 1,35 versus 1,89 .

\section{Digital prehension (precision grips)}

Figure 6 presents the results concerning the precision handling (0-5) - tip prehension (tip-to-tip pinch). The statistical evaluation demonstrates a significant difference B.Th versus A.Th. in patients of the experimental groups (E-2 and E-3).

Applying the t-test - the median value of patients before Th is 0,57 $(\mathrm{SD} 0,15)$ and A.Th. is 2,55 (SD 1,27), the t-criterion is $=-4,09$ and the $\mathrm{p}$ value $=0,001$, so we have an important statistical significance. Using the method of Mann-Whitney we received a median of Wilcoxon = 49,0 and $p$-value $p=0,001$, so statistical significance $(p<0.01)$.

If we compare the results of the experimental groups after therapy and one month later - we don't receive statistical significance $[t=0,19$; $\mathrm{p}=0,85]$, so we have a stability of the efficacy of the rehabilitation during the first 4 weeks. This stability of the effects is most expressed in the groups with mirror therapy (with grasp training). Our conclusion is that the application of functional electrical stimulations for the wrist extensors is effective (for the spasticity and for the precision grips), but is relatively instable; and for stability of the results is necessary to apply a grasp training, better with the method of mirror therapy.

The comparison of results for the experimental groups before therapy and one month after the end of the therapy demonstrates too a statistical significance $[t=-3,75 ; \mathrm{p}=0,02$, so $\mathrm{p}<0,05]$.

\section{DISCUSSION}

Our results proved the significant efficacy of complex neurorehabilitation on a lot of parameters: reduction of muscle 
Koleva I (2016) Comparative evaluation of the efficacy of different neurorehabilitation programs on the functional recovery and the autonomy of patients with post stroke hemiparesis

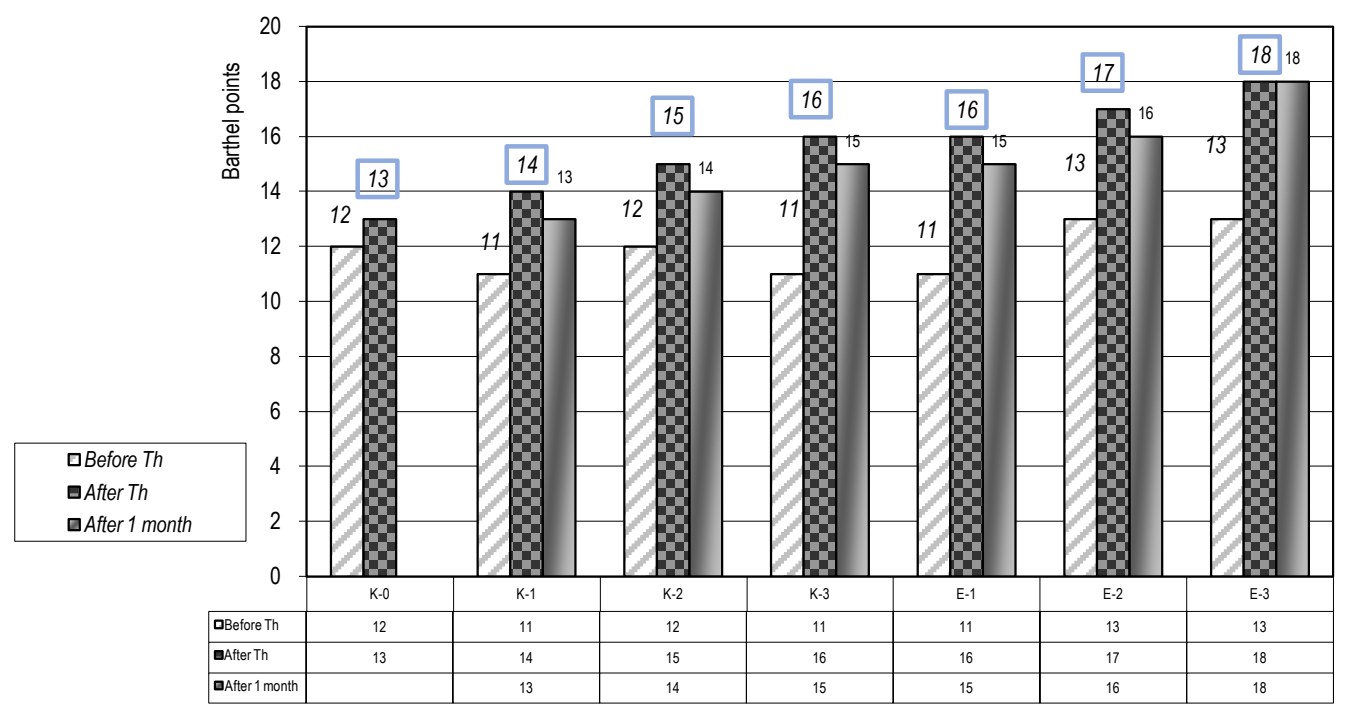

Figure 2. Functional evaluation of Barthel ADL index (0-20) - (For control and experimental groups).

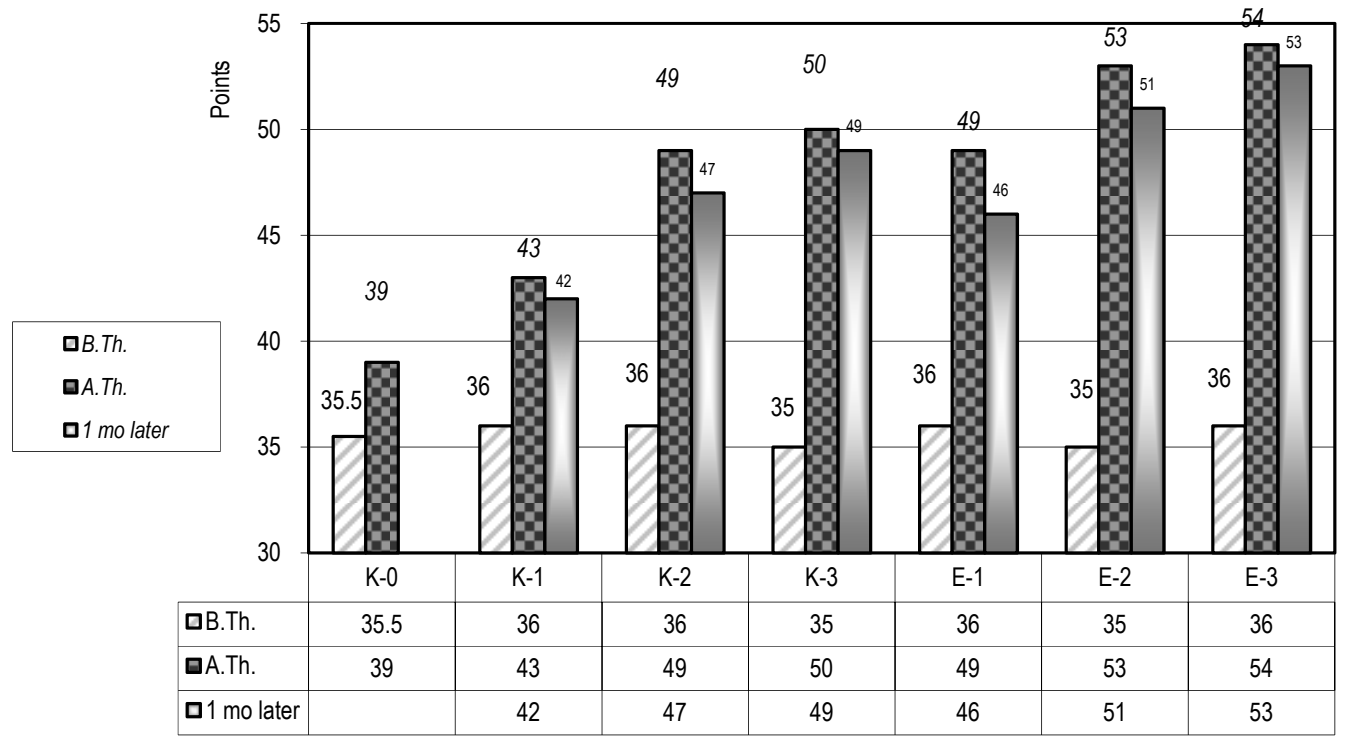

Figure 3. Evaluation of the autonomy of the paretic upper limb in ADL (test of E Michel) - (for the control and the experimental groups).

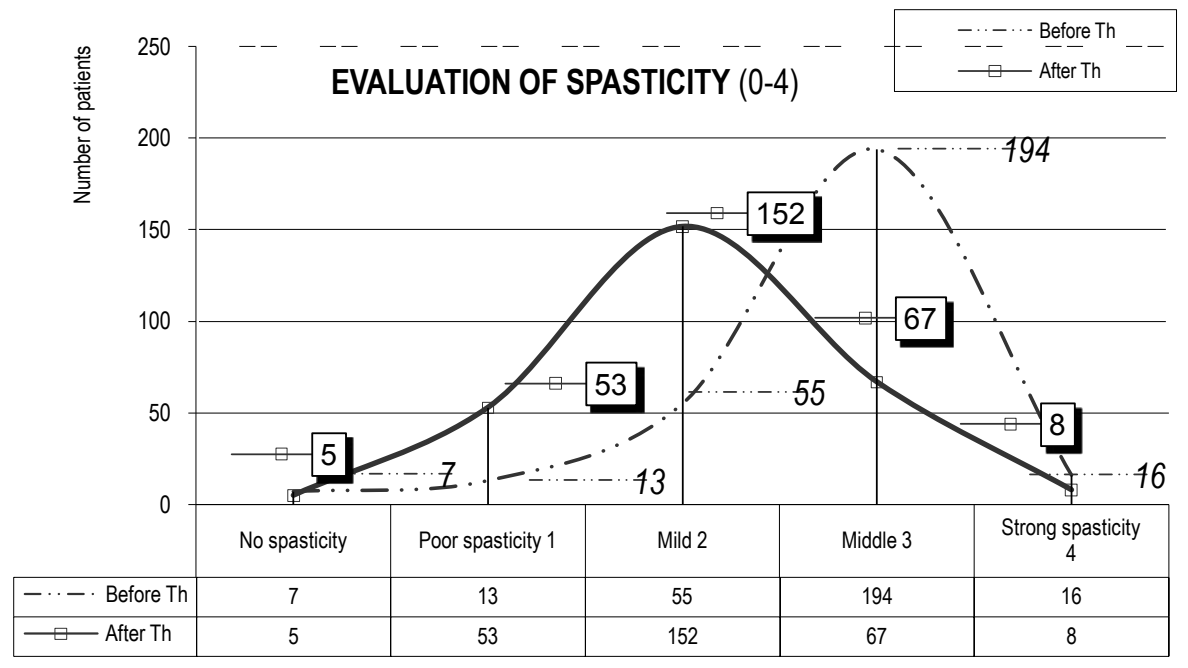

Figure 4. Evaluation of the spasticity (0-4) (in all 285 patients of the experimental groups). 
Koleva I (2016) Comparative evaluation of the efficacy of different neurorehabilitation programs on the functional recovery and the autonomy of patients with post stroke hemiparesis

Table 2. Evaluation of spasticity (0-4).

\begin{tabular}{|c|c|c|c|c|}
\hline Moment of testing & \multicolumn{2}{|c|}{ Before Th } & \multicolumn{2}{|c|}{ After Th } \\
\hline SPASTICITY & Number of patients & Percents & Number of patients & Per cents \\
\hline $0-$ no spasticity & 7 & 2,45 & 5 & 1,75 \\
\hline 1 - poor spasticity & 13 & 4,56 & 53 & 18,6 \\
\hline $2-$ mild spasticity & 55 & 19,30 & 152 & 53,33 \\
\hline 3 - middle spasticity & 194 & 68,08 & 67 & 23,51 \\
\hline 4 - strong spasticity & 16 & 5,61 & 8 & 2,81 \\
\hline TOTAL & & & & \\
\hline TOTAL & 285 & 100,00 & 285 & 100,00 \\
\hline
\end{tabular}

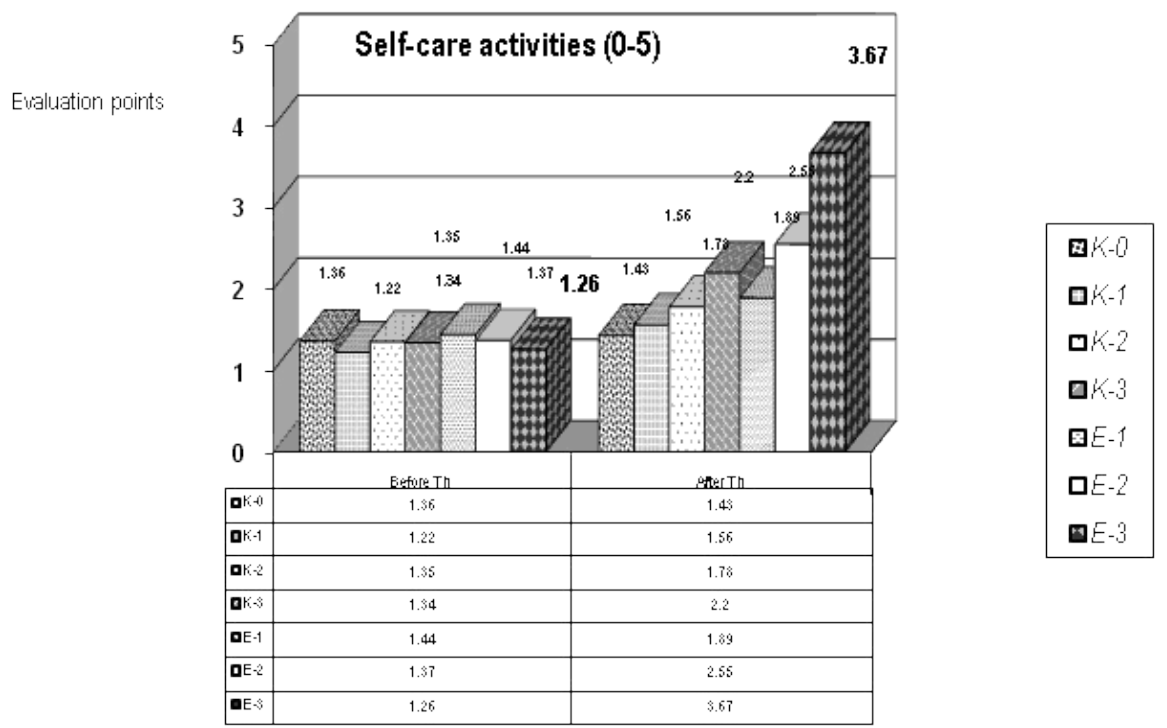

Figure 5. Evaluation of autonomy in self-care activities (0-5) (for the control and the experimental groups).
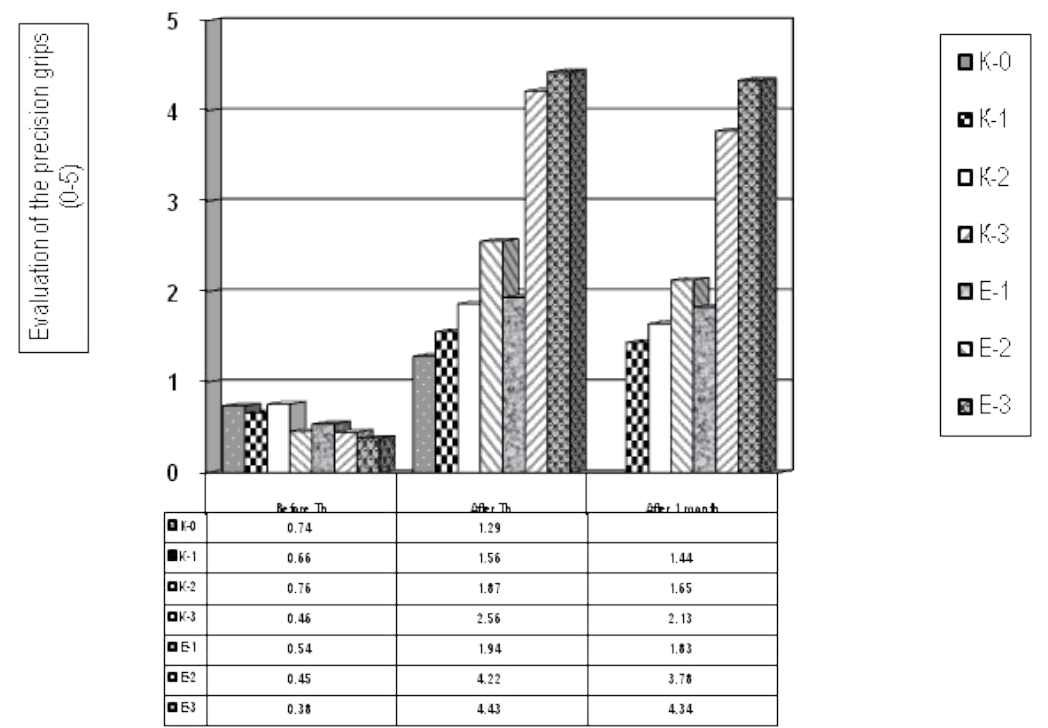

Figure 6. Evaluation of the tip prehension - precision grips (tip-to-tip pinch, scale 0-5) (for the control and the experimental groups).

weakness, increase of functional capacity of the hand (tip prehension); stabilization of the balance and locomotion (tests of Brunnstrom); improvement of the capacity of hemiparetic patients to realize main activities of self-care and some activities of domestic care.
Results of experimental groups are the most favorable, and we can emphasize the impact of electrostimulations (group E-2) and of mirror therapy for grasp training (group E-3) for acceleration of the functional recovery. 
Koleva I (2016) Comparative evaluation of the efficacy of different neurorehabilitation programs on the functional recovery and the autonomy of patients with post stroke hemiparesis

It's necessary to underline the stability of the results one month after the end of neurorehabilitation.

We agree with authors who highlight the importance of neurologic sensory-motor dysfunctions and deficits for everyday autonomy of post-stroke patients and discuss the impact of occupational therapy $[2,3,11]$. Training of basic activities of daily living (ADL - bathing, dressing, feeding, toileting, transfers) is considered as obligatory and in most reports, $47-76 \%$ of patients achieve partial or total independence in the performance of ADL, evaluated with disability rating scales. The maximal improvement is measured during the first 6 months post onset $[2,11]$.

Ultimately, the impact of mirror therapy is discussed in the literature. In a systematic review $\mathrm{H}$ Thieme et al. (2013) summarizes the effectiveness of mirror therapy for improving motor function, activities of daily living, pain, and visuospatial neglect in patients after stroke. They included randomized controlled trials and randomized crossover trials comparing mirror therapy with different interventions for patients after stroke, using relevant data of Cochrane Registers, Medline, Embase, etc. Authors analyze 14 studies with a total of 567 participants, which compared mirror therapy with other interventions. Mirror therapy was found to have a significant effect on motor function and on activities of daily living [12].

Evidence from clinical trials supports the premise that the early initiation of neurorehabilitation is crucial for the functional recovery from stroke $[2,11,14]$. When the beginning of the rehabilitation is delayed, patients may develop a lot of complications, such as muscular or joint contractures, pressure ulcers; pneumonia; urinary infections; heterotopic ossifications; depression; falls; malnutrition; deep venous thrombosis; pulmonary embolism; encephalopathy, vascular dementia $[2-4,11]$.

Mechanisms of functional recovery are discussed by a lot of authors $[2-4,11]$. Without doubt, the reduction of the extent of neurologic impairment can result from spontaneous, natural neurologic recovery (via the effects of drug treatments that limit the extent of the stroke) and from neurorehabilitation interventions that enhance neurologic functioning $[2,11]$. The training of activities of daily living (ADL), of gait and grasp can improve the quality of life of post-stroke survivors.

During the first 3 to 6 months the principal recovery mechanism is the resolution of harmful local factors: reduction of local edema, resorption of local toxins, improvement of local circulation, and recovery of partially damaged ischemic neurons. This is the cause for the early spontaneous improvement after stroke [2].

We consider that the efficacy of neurorehabilitation is due to the other mechanism of recovery - the neuroplasticity, which may continue for many months, and which can take place early or late. By definition, the brain plasticity is the ability of the nervous system to modify its structural and functional organization - principally by collateral sprouting of new synaptic connections, and by stimulation of previously latent functional pathways $[2,15]$. Other discussed mechanisms of neuroplasticity are assumption of function by undamaged, redundant neural pathways, reversibility from diaschisis, and regenerative proximal sprouting of transected neuronal axons [15]. Experimental evidence indicates that plasticity can be altered by several external factors, including pharmacologic agents, electrical stimulation, and environmental stimulation $[2,15]$.

A key aspect of neuroplasticity, very important for neurorehabilitation, is the dependence of synaptic plasticity from the functional activity $[2,16]$.

Our work demonstrates that active regular and prolonged physiotherapy and training of everyday activities (accentuating on grasp training with a mirror) promote the functional recovery and the autonomy of post-stroke hemi paretic patients, probably stimulating the brain plasticity and its functional reorganization.

Probably the stability of the effects on motor function and on autonomy in ADL (at follow-up assessment - one month after the end of rehabilitation) is due to this use-dependent neuroplasticity.

\section{CONCLUSION}

The brain has significant capacity to recover from the loss of function following a cerebral vascular accident. PRM training stimulates brain reorganization and improves functional recovery and autonomy of post-stroke patients. Systematic neurorehabilitation reduces risks of complications during the post-acute and the chronic phase, such as contractures and deconditioning [2].

The organization of neurorehabilitation is a key consideration in stroke management [17].

We agree with the White Book of PRM in Europe (2006) that the future goals for the specialty PRM cover the development of a "culture of rehabilitation" as a fundamental right for people with disabilities and one of the roles of PRM specialists is to realize that [5].

\section{REFERENCES}

1. Rehabilitation therapy after stroke. National Stroke Association. Available at: http://www.stroke.org/we-can-help/stroke-survivors/just-experienced-stroke/ rehab?pagename=REHABT. Accessed May 17, 2016.

2. Bruno-Petrina A (2016) Motor Recovery in Stroke. Medscape.

3. Mayo Clinic expert's opinion (2016) Stroke rehabilitation

4. National Institute of Neurological Disorders and Stroke (2014) Post-stroke rehabilitation.

5. Gutenbrunner C, Ward AB, Chamberlain MA (2006) White book on Physical and Rehabilitation medicine (PRM) in Europe. Produced by the Section of PRM, Union Européenne des Médecins Spécialistes (UEMS), European Board of PRM and Académie Européenne de Médecine de Réadaptation in conjunction with European Society for PRM.

6. Ward AB, Gutenbrunner C, Damjan H, Giustini A, Delarque A (2010) European Union of medical specialists (UEMS) Section of Physical \& Rehabilitation medicine: a position paper on physical and rehabilitation medicine in acute settings. $J$ Rehabil Med 42: 417-424. [Crossref]

7. Melvin JL (2001) Outcomes research in rehabilitation: scope and challenges. Am J Phys Med Rehabil 80: 78-82. [Crossref]

8. Gutenbrunner C, Meyer T, Melvin J, Stucki G (2011) Towards a conceptual description of Physical and Rehabilitation Medicine. J Rehabil Med 43: 760-764. [Crossref]

9. World Health Organization (2001). International Classification of Functioning, Disability and Health: ICF: Geneva: WHO.

10. Meyer T, Gutenbrunner C, Bickenbach J, Cieza A, Melvin J, et al. (2011) Towards a conceptual description of rehabilitation as a health strategy. $J$ Rehabil Med 43: 765769. [Crossref]

11. Koleva IB (2009) Complex neurorehabilitation algorithms for functional recovery and amelioration of independence in everyday life in socially important invalidating neurological diseases. Thesis for Doctor-es-Sciences in Physical \& Rehabilitation Medicine, Sofia. [In Bulgarian]

12. Thieme H, Mehrholz J, Pohl M, Behrens J, Dohle C (2013) Mirror therapy for improving motor function after stroke. Stroke 44: e1-2. [Crossref]

13. Brunnström, S (1970) Movement Therapy in Hemiplegia: A Neurophysiological Approach. New York: Harper \& Row. 
Koleva I (2016) Comparative evaluation of the efficacy of different neurorehabilitation programs on the functional recovery and the autonomy of patients with post stroke hemiparesis

14. Mahoney FI, Barthel DW (1965) Functional Evaluation: The Barthel Index. Md State Med J 14: 61-65. [Crossref]

15. Sala C, Cambianica I, Rossi F (2008) Molecular mechanisms of dendritic spine development and maintenance. Acta Neurobiol Exp (Wars) 68: 289-304. [Crossref]
16. Di Filippo M, Sarchielli P, Picconi B, Calabresi P (2008) Neuroinflammation and synaptic plasticity: theoretical basis for a novel, immune-centered, therapeutic approach to neurological disorders. Trends Pharmacol Sci 29: 402-412. [Crossref]

17. Kalra L, Dale P, Crome P (1993) Improving stroke rehabilitation. A controlled study. Stroke 24: 1462-1467. [Crossref]

Copyright: (C2016 Koleva I. This is an open-access article distributed under the terms of the Creative Commons Attribution License, which permits unrestricted use, distribution, and reproduction in any medium, provided the original author and source are credited. 